\title{
Evaluer la dimension d'incertitude du raisonnement clinique
}

\section{Assessing the uncertainty component of clinical reasoning}

D'une manière générale, on considère que les professionnels sont confrontés à deux types de problèmes à résoudre ${ }^{l}$. Certains problèmes, dits simples (well defined problems) ou encore bien structurés (well structured problems), sont caractérisés par le fait que l'ensemble des données nécessaires est d'emblée accessible et que la solution peut résulter d'une simple application de connaissances; ils peuvent être résolus avec un haut degré de certitude et, d'ailleurs, les professionnels experts du domaine concerné saccordent en général sur la nature de la solution correcte à mettre en ceuvre. D'autres problèmes, dits complexes (ill defined problems) ou encore mal structurés (ill structured problems), sont en revanche caractérisés par le fait que les données constitutives ne sont pas toutes disponibles d'emblée et que leur solution n'est pas univoque, impliquant une réflexion d'ordre supérieur qui requiert la mobilisation de connaissances diverses; de tels problèmes ne peuvent pas être résolus avec un haut degré de certitude et, d'ailleurs, les professionnels experts du domaine concerné sont souvent en désaccord quant à la meilleure solution à mettre en ouvre, y compris a posteriori quand le problème peut être considéré comme ayant été résolu.

De façon spécifique, il est reconnu que les problèmes auxquels sont confrontés les professionnels de santé sont, dans l'immense majorité des cas, de type complexe ou mal structuré. Le raisonnement clinique, considéré au sens générique du terme-c'est-à-dire en y incluant à la fois le processus de raisonnement diagnostique et celui de prise de décisions d'investigation et de traitement-, désigne le processus de résolution de problèmes que les cliniciens mettent en ouvre face à de tels problèmes ${ }^{2}$. Il n'est donc guère surprenant de constater que, face à un même problème de santé, les chemins empruntés pour élaborer les solutions varient selon les cliniciens, y compris au sein d'une même discipline.

Selon Schön ${ }^{3}$, les institutions d'enseignement supérieur entraînent bien leurs étudiants à la résolution des problèmes de structure simple mais mal à celle des problèmes de structure complexe. La même remarque peut s'appliquer à l'évaluation du raisonnement clinique. Nous disposons de bons outils pour évaluer la résolution de problèmes cliniques de structure simple et de peu d'outils pour évaluer celle des problèmes de structure complexe, notamment pour ceux qui impliquent la prise de décision en contexte d'incertitude, caractéristique clé de l'exercice professionnel dans les sciences de la santé.

Si ces prémisses sont exactes, un outil d'évaluation $d u$ raisonnement clinique qui sonde la capacité à résoudre les problèmes de structure simple devrait être régulièrement en défaut pour déceler les personnes réellement compétentes dans un domaine de raisonnement; en effet, des étudiants qui ont accumulé beaucoup de connaissances, déclaratives en particulier, peuvent mieux réussir à ces tests que des praticiens, dont les savoirs livresques sont moins disponibles, mais qui sont riches d'expériences cliniques et qui ont, de ce fait, construit de riches et pertinents réseaux de connaissances d'action. La littérature désigne cela sous le terme d'effet intermédiaire de l'évaluation: les cliniciens de niveau intermédiaire, des résidents ou internes en fin de formation par exemple, obtiennent régulièrement à ce type de tests des scores supérieurs à ceux des praticiens expérimentés. À l'inverse, sil était établi que des praticiens possédant plusieurs années d'expérience obtenaient régulièrement de meilleurs scores à tel ou tel test d'évaluation du raisonnement clinique, cela constituerait un argument fort en faveur de la validité de ces tests, c'est-à-dire leur capacité à bien mesurer ce qu'ils prétendent mesurer, en l'espèce la qualité du raisonnement clinique.

Les questions à choix multiples (QCM) portant sur des cas cliniques, dits à contexte riche, sont tenues pour être actuellement un standard en matière d'évaluation du raisonnement clinique ${ }^{4}$. Elles présentent un cas clinique pour lequel le candidat doit synthétiser l'information disponible, toutes les données nécessaires à la solution du problème étant fournies; par construction, il n'existe qu'une seule bonne réponse. Ces examens permettent une correction automatisée et peuvent être administrés à de très grands groupes d'étudiants, d'où leur large utilisation dans les examens de certification, aux États-Unis notamment.

Le test de concordance de script (TCS) est un format de test plus récent, qui cherche à prendre en compte la dimension d'incertitude inhérente au processus de raisonnement clinique ${ }^{4}$. Il présente un cas clinique pour lequel toute l'information clinique nécessaire à sa résolution n'est pas fournie ou dans lequel certaines 
données sont ambiguës. Dans de tels contextes, nous savons que les cliniciens experts adoptent des cheminements de raisonnement variables. Il ne peut donc y avoir une seule "bonne réponse ". Cette variabilité est incorporée dans le processus d'attribution du score, qui pondère les indices numériques affectés à chacune des réponses proposées à l'étudiant en fonction des proportions respectives d'experts ayant sélectionné celles-ci. Une telle procédure permet de dépasser l'appréciation binaire en " tout ou rien".

L'étude présentée dans ce numéro par Jean-Pierre Fournier et ses collaborateurs ${ }^{5}$ compare les résultats obtenus à ces deux formats de tests par plusieurs populations de médecins, de niveaux d'expérience différents, travaillant en salle d'urgence. Les deux tests ont été réalisés avec soin comme en témoigne les excellentes valeurs de leurs coefficients respectifs de cohérence interne (mesurées par le test alpha de Cronbach). Les résultats montrent une disparition de l'effet intermédiaire. Les médecins les plus expérimentés obtiennent de meilleurs scores que les médecins de niveau intermédiaire (les internes). Ce travail est le premier à démontrer une telle aptitude discriminante dans la littérature internationale. Par ailleurs, les scores obtenus aux TCS et aux QCM varient dans le même sens mais sans corrélation significative. Ce point indique que QCM et TCS explorent deux aspects distincts mais complémentaires de la compétence au raisonnement clinique. Ces résultats devront être confirmés par d'autres études et dans d'autres disciplines mais, s'ils étaient confirmés, ils suggéreraient que le TCS peut légitimement revendiquer une originalité et une valeur ajoutée au sein des outils disponibles pour l'évaluation du raisonnement clinique. Tout en respectant les exigences de la perspective docimologique dans laquelle il sinscrit, et à côté d'autres outils éprouvés, qui mesurent déjà les dimensions les moins incertaines et les moins ambiguës du raisonnement clinique, tels que les QCM à contexte riche, il pourrait compléter avec profit les moyens disponibles ou en développement, qui cherchent à appréhender la dimension d'incertitude propre au processus de raisonnement clinique. Quand on connaît les conséquences quiont les procédés d'évaluation à l'égard des apprentissages des étudiants -et dont rend compte, par exemple, l'adage "l'évaluation conduit le curriculum " -, l'introduction d'un tel outil serait de nature à orienter favorablement les activités d'enseignement et d'apprentissage, au service d'une meilleure préparation des futurs professionnels à la dimension d'incertitude du raisonnement clinique et à la réalité de la pratique clinique.
Il reste que les problèmes que soulève le souhait de prendre authentiquement en compte la dimension d'incertitude du raisonnement clinique ne sont pas tous résolus. Dès lors que l'on reconnaît qu'il y a, en effet et presque toujours, plusieurs manières de résoudre avec compétence un problème de santé donné, notre rôle d'enseignant et d'évaluateur devrait être d'aider les étudiants à être en mesure d'élaborer et de justifier l'une des solutions raisonnables et acceptables possibles. Dès lors, un étudiant qui propose et argumente correctement l'une de ces solutions ne devrait pas être pénalisé dans l'attribution des scores, au seul motif qu'une minorité parmi les experts a choisi cette solution. Bien évidemment, les outils d'évaluation qui permettraient de dépasser ce point ont d'autres inconvénients ou limites : lourdeur, correction non automatisable, nécessité de sinscrire dans une démarche plus qualitative pour apprécier les critères classiques de validité et de fidélité, etc. A l'évidence, la recherche en éducation des sciences de la santé a encore des pistes fécondes à explorer en matière d'évaluation du raisonnement clinique.

Bernard CHARLIN Mailto:bernard.charlin@umontreal.ca

\section{Références}

1. King PM, Kitchener KS. Developping reflective judgment : Understanding and promoting intellectual growth and critical thinking in adolescents and adults. San Francisco (CA): JosseyBass, 1994.

2. Nendaz M, Charlin B, LeBlanc V, Bordage G. Le raisonnement clinique : données issues de la recherche et implications pour l'enseignement. Pédagogie Médicale 2005;6:234-53

3. Schön D. The Reflective Practitioner: How Professionals Think in Action. New York: Basic Books, 1983.

4. Charlin B, Bordage G, Van der Vleuten C. L'évaluation du raisonnement clinique. Pédagogie Médicale 2003;4:42-52.

5. Fournier J-P, Thiercelin D, Pulcini C et al. Évaluation $d u$ raisonnement clinique en médecine d'urgence : les tests de concordance des scripts décèlent mieux l'expérience clinique que les questions à choix multiples à contexte riche. Pédagogie Médicale, 2006,7:20-30. 